\title{
Half dose of TauroLock; does it work?
}

\author{
Sima Golmohamadi, Hamid Reza Omrani*, Nadia Asadi, Elaheh Sharifi, Mohammad Reza Zandkarimi \\ Nephrology Ward and Immunology Department, Kermanshah University of Medical Sciences, Kermanshah, Iran
}

\section{A R T I C L E I N F O}

Article Type:

Original

\section{Article History:}

Received: 28 January 2018

Accepted: 10 May 2018

Published online: 1 June 2018

\section{Keywords:}

Taurolock

Catheter related bacteremia

Hemodialysis

End-stage renal disease

\begin{abstract}
A B S T R A C T
Introduction: Bacteremia is one of the most important complications in central vein catheter used in hemodialysis. TauroLock is known as an effective catheter lock solution (antimicrobial plus anticoagulant solution) in preventing catheter-related bacteremia (CRB). However, its cost is high and not covered by the most insurance companies.

Objectives: The aim of this study is to investigate the effect of the medication as a half dose for its economic advantages.

Patients and Methods: In this clinical trial study, 152 patients were divided into case $(n=62)$ and control groups $(\mathrm{n}=90)$. In the case group, for 1 year, TauroLock and heparin were injected into the catheter's lumen (equal dose), but in the control group, only heparin was injected. For detecting bacteremia, blood cultures were obtained.

Results: According to the results, there was a significant difference in CRB between the two groups, at the base time, 3, 6 and 12 months after injection of TauroLock $(P<0.05)$. There was a significant difference in $\mathrm{CRB}$, at the base time, 3, 6 and 12 months, after injection of TauroLock for the case group $(P<0.05)$, but there was no significant difference for the control group $(P<0.05)$. A positive effect in decreasing CRB was seen in the patients, based on age, sex, BMI, duration of hemodialysis, level of serum albumin, diabetes mellitus history and serum ferritin.

Conclusion: The catheter lock solution TauroLock, even in half dose, after each hemodialysis session, could significantly decrease the rate of CRB in hemodialysis patients
\end{abstract}

Implication for health policy/practice/research/medical education:

Usage of TauroLock as an antimicrobial drug that prevents CRB has been recommended in most studies, but the high cost of the drug prevents its widespread use in hemodialysis patients. Therefore, in this study the effect of TauroLock as a half dose for its economic advantages was investigated.

Please cite this paper as: Golmohamadi S, Omrani HR, Asadi N, Sharifi E, Zandkarimi MR. Half dose of TauroLock; does it work? J Renal Inj Prev. 2018;7(3):180-185. doi: 10.15171/jrip.2018.43.

\section{Introduction}

Hemodialysis is the most common renal replacement therapy in end-stage renal disease (ESRD). Vascular access is required to conduct hemodialysis and one of the most predominant ways is central venous catheter (CVC), while heparin lock is used to prevent thrombosis in catheters (1). Applying central venous catheter increases catheter-related bacteremia (CRB). A report stated that CRB rate was 2.5-5.5 times for every 1000-day dialysis that is equivalent to $0.9-2$ times in performing one-year dialysis (2). CRB occurrence is related to the time distance between using catheters. In one study CRB has been reported 35\% within 3 months and $48 \%$ during 6 months of catheter insertion (3). This infection increases mortality, morbidity and extra costs for health care system. CRB may be predisposed to metastatic infections like osteomyelitis, endocarditis, septic arthritis and epidural abscess in 5\%-10\% of patients with catheter insertion $(2,3)$. In fact, using catheters increasingly results in metastatic infections (4). The most common risk factor for CRB occurrence is the time of applying catheter. Other risk factors include recent surgery, diabetes mellitus, iron overload, immunosuppression and hypoalbuminemia $(5,6)$. Using antibiotic lock solutions for preventing CRB is effective, especially in patients who are high risk. In a meta-analysis, it was detected that this method had a high 
risk of antibiotic resistance $(7,8)$. TauroLock (Taurolidine and $4 \%$ citrate) was used in several studies while a metaanalysis that indicated its effectiveness and accordingly no side effects were reported $(9,10)$.

It seems that the catheters locked by Taurolidine are an effective and safe way to reduce catheter-caused septicemia. Taurolidine contains a simple and general antibacterial effect. In vitro examinations with subinhibitory doses of Taurolidine did not cause the microorganism resistance (11). Therefore, it is unlikely that long-time TauroLock injection in dialysis line results to increase bacterial infections resistant to the drug. No side effects were reported for TauroLock, and this compound is quickly metabolized to Taurine and carbon dioxide. Using Taurolidine for a long-time is possible with no toxicity and its anti-adherence property results in protecting against bacteria and fungi adhering to central venous catheter (12).

\section{Objectives}

In this study, the preventive effect of the drug in patients with catheter in dialysis center of Imam Khomeini and Imam Reza hospitals in Kermanshah were assessed. For its economic advantages, TauroLock was used to fill a half catheter lumen (half dose) after each dialysis session, and the rest of the catheter lumen space was filled with heparin, by the impression that by a suitable response to this drug with half dose, we can prevent CRB occurrence at a lower cost, which is beneficial modality.

\section{Patients and Methods}

\section{Study population}

During 1 year, 152 patients including 62 in the case group (40.8\%) and 90 in the control group (59.2\%) were considered. Variables were as follows; age, gender, body mass index (BMI), time of using catheter (less or more than one year), using TauroLock, catheter infection, diabetes mellitus history, iron overload (ferritin $>300 \mathrm{ng} /$ $\mathrm{mL}$ ), CRP and hypoalbuminemia (albumin $>4 \mathrm{~g} / \mathrm{dL}$ ). The symptoms of CRB included fever and chills during dialysis, in the absence of other causes. For this category of patients, two blood cultures from catheters were done to the time interval of fifteen minutes. If the cultures were positive, $\mathrm{CRB}$ would be confirmed. The information about patients like demographic data was analyzed in SPSS.

Inclusion criteria were; patient under hemodialysis by catheter in dialysis center of Imam Khomeini and Imam Reza hospitals. They agreed to participate in this study. Accordingly, exclusion criteria were; IV drug abuse, changing dialysis from catheter to AVF, patients with surgical history and immunosuppression.

After choosing patients, they were assimilated based on field variables. Causal layered analysis was conducted when they were not assimilated by goals.

\section{Ethical issues}

The research followed the tenets of the Declaration of Helsinki. The study was approved by the institutional ethics committee of Kermanshah University of Medical Sciences and was registered in Iranian Registry of Clinical Trials (identifier: IRCT2016020221066N2; http://www. irct.ir/trial/18554). Written informed consent was obtained from all participants too.

\section{Data analysis}

Quantitative data were reported by mean \pm standard deviation (SD) and qualitative data by percentage and frequency. Two groups were compared based on MannWhitney $U$ and chi-square tests and were checked for assimilation by chi-square and independent $t$ test. The significance level was 0.05 . The data were analyzed by SPSS, version 22. Mann-Whitney U test was applied to compare the intervention and control groups for CRB, levels CRP, albumin level and ferritin level in dialysis patients with catheter during base time, 3, 6 and 12 months after injection of TauroLock. Chi-square test was applied to compare the intervention with the control group for CRB in dialysis patients with catheter based on age, gender, dialysis time, and diabetes mellitus history, level of serum albumin and ferritin.

\section{Results}

The results of descriptive properties and comparing age, gender, body mass index (BMI), time of using catheter and diabetes mellitus between case and control groups were shown in Table 1 . There was no significant difference in mentioned terms between two groups $(P>0.05)$. Table 2 shows frequencies and comparing CRP and CRB in

Table 1. Descriptive properties and comparing age, sex, Body Mass Index (BMI), time of using catheter, diabetes mellitus history and bacteremia history resulted from catheter in dialysis patients of case and control groups

\begin{tabular}{|c|c|c|c|c|}
\hline \multirow{2}{*}{ Feature } & & \multicolumn{2}{|c|}{ Group } & \multirow{2}{*}{$P$ value } \\
\hline & & Case & Control & \\
\hline Age & Mean \pm SD & $61.03 \pm 16.91$ & $55.23 \pm 18.43$ & 0.205 \\
\hline \multirow{2}{*}{ Sex } & Male, No. \% & $32(51.6)$ & $63(70.0)$ & \multirow{2}{*}{0.192} \\
\hline & Female, No. \% & $30(48.4)$ & $27(30.0)$ & \\
\hline BMI & Mean \pm SD & $22.48 \pm 4.46$ & $24.17 \pm 4.27$ & 0.115 \\
\hline Duration of use of catheter & Mean \pm SD & $11.9 \pm 30.81$ & 27.6045 .17 & 0.718 \\
\hline \multirow{2}{*}{ Diabetes mellitus history } & Yes, No. \% & $18(29.0)$ & $27(30.0)$ & \multirow{2}{*}{1} \\
\hline & No, No. \% & $44(71.0)$ & $63(70.0)$ & \\
\hline
\end{tabular}


dialysis patients with catheter in case and control groups during base time, 3, 6 and 12 months after injection of TauroLock. Intergroup comparison showed a significant difference in $6(P=0.046)$ and 12 months $(P=0.002)$ between two groups for CRP. Therefore the frequency of $\mathrm{CRP}$ in the case group was less than the control group. In addition, intergroup comparison showed a significant difference in $3(P=0.018), 6(P=0.011)$ and 12 months $(P=0.001)$ between two groups for CRB; therefore, the frequency of CRP in the case group was less than the control group. Intragroup comparison showed that changings of frequency of CRP and CRB during time had just a significant difference in case group $(P=0.024$ and $P=0.002$, respectively) that the frequency with time had a reverse correlation.

Table 3 shows descriptive properties and comparing level of albumin and ferritin in two groups during base time, 3 , 6 and 12 months after injection of TauroLock. Intergroup comparison showed no significant difference all times between two groups $(P>0.05)$ for albumin and ferritin levels. Intragroup comparison showed just the changes of albumin level in case group during time had a significant difference $(P=0.027)$ with a direct relationship.
Frequencies and comparing CRB in dialysis patients with catheter in two groups based on age, gender and BMI were shown in Table 4 . There was a difference for CRB frequency between two groups in terms of age $(<50$ and $>50$ years: $\mathrm{P}=0.037$ and $P=0.008$ respectively), gender (male and female: $P=0.002$ and $P=0.430)$, and BMI $(<25$ and $>25 \mathrm{~kg} / \mathrm{m}^{2} ; P=0.035$ and 0.014 respectively).

Frequencies and comparing CRB in dialysis patients with catheter in two groups based on dialysis time, diabetes, serum albumin and serum ferritin levels were shown in Table 5. There was a difference for CRB frequency between two groups in terms of time of dialysis $(<1$ and $>1$ years: $P=0.010$ and $P=0.033$ respectively), diabetes (yes and no: $\mathrm{P}=0.031$ and $P=0.014$ respectively), base albumin level ( $<4$ and $>4 \mathrm{~g} / \mathrm{dL} ; P=0.015$ and 0.047 respectively), and base ferritin level $(<300$ and $>300 \mathrm{ng} / \mathrm{mL} ; P=0.033$ and $P=0.004$ respectively).

\section{Discussion}

There was no significant difference among age, gender, BMI, time of using catheter and history of diabetes mellitus between the case and control groups. According to the most significant results, injecting TauroLock - with

Table 2. Frequencies and comparing CRP and CRB in dialysis patients with catheter in case and control groups during base time, 3,6 and 12 months after injection of TauroLock

\begin{tabular}{|c|c|c|c|c|c|c|}
\hline \multirow{2}{*}{ Variable } & \multirow{2}{*}{ Measuring time } & \multicolumn{3}{|l|}{ Group } & \multicolumn{2}{|c|}{ Intergroup comparisons } \\
\hline & & Case, No. (\%) & & Control, No. (\%) & Testing statistics & $P$ value \\
\hline \multirow[t]{6}{*}{ CRP } & 0 & $20(32)$ & & 35 (39) & 0.89 & 0.3734 \\
\hline & 3 months & $16(26)$ & & 35 (39) & 1.71 & 0.0872 \\
\hline & 6 months & $13(19)$ & & $30(33.3)$ & 2 & 0.046 \\
\hline & 12 months & $6(9.7)$ & & $25(27.7)$ & 2.98 & 0.002 \\
\hline & Intergroup comparisons & Testing statistics & 9.45 & 3.76 & & \\
\hline & & $P$ value & 0.024 & 0.288 & & \\
\hline \multirow[t]{5}{*}{ CRB } & 3 months & $10(16.1)$ & & $27(30.0)$ & 2.08 & 0.018 \\
\hline & 6 months & $8(17.8)$ & & $28(31.1)$ & 6.73 & 0.011 \\
\hline & 12 months & $6(9.7)$ & & $30(33.3)$ & 11.36 & 0.001 \\
\hline & Intergroup comparisons & Testing statistics & 12 & 0.483 & & \\
\hline & & $P$ value & 0.002 & 0.786 & & \\
\hline
\end{tabular}

Table 3. Descriptive properties and comparing level of albumin and ferritin in case and control groups during base time, 3,6 and 12 months after injection of TauroLock

\begin{tabular}{|c|c|c|c|c|c|c|}
\hline \multirow[t]{2}{*}{ Variable } & \multirow[t]{2}{*}{ Measuring time } & \multicolumn{3}{|l|}{ Group } & \multicolumn{2}{|c|}{ Intergroup comparisons } \\
\hline & & Case, Mean \pm SD & & Control, Mean \pm SD & Testing statistics & $P$ value \\
\hline \multirow[t]{6}{*}{ Albumin } & 0 & $3.62 \pm 0.25$ & & $3.52 \pm 0.4$ & 1.1 & 0.27 \\
\hline & 3 months & $3.67 \pm 0.38$ & & $3.6 \pm 0.38$ & 0.536 & 0.592 \\
\hline & 6 months & $3.8 \pm 0.43$ & & $0.68 \pm 0.38$ & 0.106 & 0.106 \\
\hline & 12 months & $3.75 \pm 0.38$ & & $3.72 \pm 0.3$ & 0.547 & 0.584 \\
\hline & Intergroup & Testing statistics & 9.15 & 4.13 & & \\
\hline & comparisons & $P$ value & 0.027 & 0.248 & & \\
\hline \multirow[t]{6}{*}{ Ferritin } & 0 & $377 \pm 209.01$ & & $312.16 \pm 100.54$ & 0.859 & 0.391 \\
\hline & 3 months & $358.9 \pm 145.01$ & & $324.16 \pm 93.37$ & 1.108 & 0.272 \\
\hline & 6 months & $383.37 \pm 236.44$ & & $349.33 \pm 83.23$ & 0.57 & 0.569 \\
\hline & 12 months & $399.19 \pm 324.31$ & & $356.06 \pm 90.71$ & 0.902 & 0.367 \\
\hline & Intergroup & Testing statistics & 3.68 & 2.93 & & \\
\hline & comparisons & $P$ value & 0.298 & 0.051 & & \\
\hline
\end{tabular}


Table 4. Frequencies and comparing CRB in dialysis patients with catheter in case and control groups based on age, sex and BMI

\begin{tabular}{|c|c|c|c|c|c|}
\hline \multirow{2}{*}{ Variable } & \multirow{2}{*}{ Feature } & \multirow{2}{*}{ CRB } & \multicolumn{2}{|c|}{ Group } & \multirow{2}{*}{$P$ value } \\
\hline & & & Case, No. (\%) & Control, , No. (\%) & \\
\hline \multirow{4}{*}{ Age (y) } & \multirow{2}{*}{ Less than 50} & Yes & $0(0.0)$ & $8(28.6)$ & \multirow{2}{*}{0.037} \\
\hline & & No & $14(100)$ & $20(71.4)$ & \\
\hline & \multirow{2}{*}{ More than 50} & Yes & $6(12.5)$ & $22(35.5)$ & \multirow{2}{*}{0.008} \\
\hline & & No & $62(87.5)$ & $40(64.5)$ & \\
\hline \multirow{4}{*}{ Sex } & \multirow{2}{*}{ Male } & Yes & $0(0.0)$ & 23 (31.9) & \multirow{2}{*}{0.002} \\
\hline & & No & $19(100)$ & $49(68.1)$ & \\
\hline & \multirow{2}{*}{ Female } & Yes & $6(14.0)$ & $7(38.9)$ & \multirow{2}{*}{0.43} \\
\hline & & No & $37(86.0)$ & $11(61.1)$ & \\
\hline \multirow{4}{*}{ BMI } & \multirow{2}{*}{ Less than 25} & Yes & $2(6.3)$ & $8(28.6)$ & \multirow{2}{*}{0.035} \\
\hline & & No & $30(93.8)$ & $20(71.4)$ & \\
\hline & \multirow{2}{*}{ More than 25} & Yes & $4(13.3)$ & $22(39.3)$ & \multirow{2}{*}{0.014} \\
\hline & & No & $26(86.7)$ & $34(60.7)$ & \\
\hline
\end{tabular}

Table 5. Frequencies and comparing CRB in dialysis patients with catheter in case and control groups based on dialysis time, diabetes, level of serum albumin and level of serum ferritin

\begin{tabular}{|c|c|c|c|c|c|}
\hline \multirow{2}{*}{ Variable } & \multirow{2}{*}{ Feature } & \multirow{2}{*}{ CRB } & \multicolumn{2}{|c|}{ Group } & \multirow{2}{*}{$P$ value } \\
\hline & & & Case, No. (\%) & Control, , No. (\%) & \\
\hline \multirow{4}{*}{ Time of dialysis } & \multirow{2}{*}{ Less than 1 year } & Yes & $4(8.3)$ & $20(28.2)$ & \multirow{2}{*}{0.01} \\
\hline & & No & $44(91.7)$ & $51(71.8)$ & \\
\hline & \multirow{2}{*}{ More than 1 year } & Yes & $2(14.3)$ & $10(52.6)$ & \multirow{2}{*}{0.033} \\
\hline & & No & $12(85.7)$ & $9(47.4)$ & \\
\hline \multirow{4}{*}{ Diabetes } & \multirow{2}{*}{ Yes } & Yes & $2(10.5)$ & $13(39.4)$ & \multirow{2}{*}{0.031} \\
\hline & & No & $17(89.5)$ & $20(60.6)$ & \\
\hline & \multirow{2}{*}{ No } & Yes & $4(9.3)$ & $17(29.8)$ & \multirow{2}{*}{0.014} \\
\hline & & No & $39(90.7)$ & $40(70.2)$ & \\
\hline \multirow{4}{*}{ Level of base albumin (mg/dL) } & \multirow{2}{*}{ Less than 4} & Yes & $4(9.5)$ & $19(31.1)$ & \multirow{2}{*}{0.015} \\
\hline & & No & $38(90.5)$ & $42(68.9)$ & \\
\hline & \multirow{2}{*}{ More than 4} & Yes & $2(10.0)$ & 11 (37.9) & \multirow{2}{*}{0.047} \\
\hline & & No & $18(90.0)$ & $18(62.1)$ & \\
\hline \multirow{4}{*}{ Level of base ferritin } & \multirow{2}{*}{ Less than 300} & Yes & $6(17.6)$ & $20(40.0)$ & \multirow{2}{*}{0.033} \\
\hline & & No & $28(82.4)$ & $30(60.0)$ & \\
\hline & \multirow{2}{*}{ More than 300} & Yes & $0(0.0)$ & $10(25.0)$ & \multirow{2}{*}{0.004} \\
\hline & & No & $28(100.0)$ & $30(75.0)$ & \\
\hline
\end{tabular}

half dose - and heparin decreased CRB in dialysis patients with catheter. The positive effects of compounding TauroLock (half dose) and heparin on decreasing CRB were observed in patients with following features; less or more than 50 years old age, male or female, with BMI less or more than 25 , dialysis time less or more than one year, with or without diabetic history, with albumin level less or more than $4 \mathrm{~g} / \mathrm{dL}$ and ferritin level less or more than 300 $\mathrm{ng} / \mathrm{mL}(P<0.05)$. Therefore, age, gender, BMI, duration of dialysis, duration of use of catheter, diabetes mellitus history, levels of albumin and ferritin had no effect on the performance of TauroLock.

To the best of our knowledge, no study investigated the effect of TauroLock -with half dose- and heparin on CRB in hemodialysis patients with catheter. But in recent studies, the positive effect of TauroLock on catheter related infections was reported among dialysis patients $(13,14)$. Some studies have shown the prevention effect of TauroLock in CRB among hemodialysis patients. Al Malki et al found that compounding TauroLock and heparin prevents CRB in hemodialysis patients (15). Fontseré et al stated that compounding TauroLock and heparin is effective in decreasing CRB in hemodialysis patients with catheter (16). Stein et al concluded that $\mathrm{CRB}$ in hemodialysis patients receiving TauroLock and heparin is less than patients who received just heparin (17). Similarly, Zwiech et al found that compounding TauroLock and heparin, compared to heparin, affected decreasing CRB among hemodialysis patients with catheter (18). Furthermore, Solomon et al concluded that adding $500 \mathrm{U} / \mathrm{mL}$ heparin to TauroLock can decrease the need for thrombolysis without increasing bacteremia (19). Liu et al found that antimicrobial solution like TauroLock with heparin could be the best preventative factor for CRB in hemodialysis patients (20). Likewise, Murray et al reported that using throline-citrate - heparin instead of heparin can be related to central venous catheter, as the catheter lock solution with $56 \%$ decrease in hemodialysis related infections (21). The results of this study are compatible with other mentioned studies. 
Kavosi et al, in a meta-analysis, found that CRB in hemodialysis patients who used TauroLock, was less than hemodialysis patients who used heparin. However, they showed an unequal result that, TauroLock in comparison to the combination of heparin and gentamicin is not the preferable strategy to prevent CRB than TauroLock alone (22).

Solomon et al found no difference between bacteremia episodes in hemodialysis patients who used throlinecitrate and who received heparin. In fact, using throline-citrate does not decrease bacteremia and needs thrombolytic therapy (23). The densities of throline, applied for lock solutions, are $1.35 \%$ to $2 \%$. Still no studies have reported throline decrease in lumen catheter or the relationship between throline density and CRB (24).

\section{Conclusion}

According to the findings, medication of half-dose TauroLock, after each dialysis session, can decrease CRB among dialysis patients with central catheter. Under the positive effect of TauroLock in preventing catheter-related infections in dialysis patients, prescribing it beside other effective drugs in hemodialysis patients with catheter could be recommended.

\section{Limitations of the study}

Given the contradicting results of this and other studies, larger-scale studies are recommended to be conducted on the long-term effects of contrast agents on renal function.

\section{Acknowledgments}

This article is part of an M.D thesis by Elahe Sharifi (\# 95025), approved by the Research Deputy of Kermanshah University of Medical Sciences. The authors wish to thank all staffs of the Medical ward of Emam Reza Hospital for their cooperation in this study.

\section{Authors' contribution}

SG conducted the research and contributions to the conception and design of the research. HRO prepared the primary draft. NA contributed to the acquisition of data. ES contributed to the analysis of data. MRZ contributed to the drafting of the manuscript and final approval of the manuscript.

\section{Conflicts of interest}

The authors declare that they have no competing interest.

\section{Ethical considerations}

Ethical issues (including plagiarism, misconduct, data fabrication, falsification, double publication or submission, redundancy) have been completely observed by the authors.

\section{Funding/Support}

This study was supported by the Research Deputy of Kermanshah University of Medical Sciences.

\section{References}

1. Hemodialysis Adequacy 2006 Work Group. Clinical practice guidelines and clinical practice recommendations 2006 updates hemodialysis adequacy peritoneal dialysis adequacy vascular access. Am J Kidney Dis. 2006;48:S2-90. doi: 10.1053/j.ajkd.2006.03.051.

2. Allon M. Dialysis catheter-related bacteremia: treatment and prophylaxis. Am J Kidney Dis. 2004;44:779-91.

3. Lee T, Barker J, Allon M. Tunneled catheters in hemodialysis patients: reasons and subsequent outcomes. Am J Kidney Dis. 2005; 46:501-8. doi: 10.1053/j.ajkd.2005.05.024.

4. Kovalik EC, Raymond JR, Albers FJ, Berkoben M, Butterly DW, Montella B, et al. A clustering of epidural abscesses in chronic hemodialysis patients: risks of salvaging access catheters in cases of infection. J Am Soc Nephrol. 1996;7:2264-7.

5. Teehan GS, Bahdouch D, Ruthazer R, Balakrishnan VS, Snydman DR, Jaber BL. Iron storage indices: novel predictors of bacteremia in hemodialysis patients initiating intravenous iron therapy. Clin Infect Dis. 2004;38:1090-4.

6. Tanriover B, Carlton D, Saddekni S, Hamrick K, Oser $\mathrm{R}$, Westfall $\mathrm{AO}$, et al. Bacteremia associated with tunneled dialysis catheters: comparison of two treatment strategies. Kidney Int. 2000;57:2151. doi: 10.1046/j.15231755.2000.00067.x.

7. Yahav D, Rozen-Zvi B, Gafter-Gvili A, Leibovici L, Gafter U, Paul M. Antimicrobial lock solutions for the prevention of infections associated with intravascular catheters in patients undergoing hemodialysis: systematic review and meta-analysis of randomized, controlled trials. Clin Infect Dis. 2008;47:83-93. doi: 10.1086/588667.

8. O'Horo JC, Silva GL, Safdar N. Anti-infective locks for treatment of central line-associated bloodstream infection: a systematic review and meta-analysis. Am J Nephrol. 2011;34:415-22. doi: 10.1159/000331262.

9. Gorman SP, McCafferty DF, Woolfson AD, Jones DS. Reduced adherence of micro-organisms to human mucosal epithelial cells following treatment with Taurolin, a novel antimicrobial agent. J Appl Bacteriol. 1987;62:315-20.

10. Geron R, Tanchilevski O, Kristal B. [Catheter lock solution-taurolock for prevention of catheter-related bacteremia in hemodialysis patients]. Harefuah. 2006;145:881-4.

11. Torres-Viera C, Thauvin-Eliopoulos C, Souli M, DeGirolami P, Farris MG, Wennersten CB, et al. Activities of taurolidine in vitro and inexperimental enterococal endocarditis. Antimicrob Agents Chemother. 2000;44:1720-4.

12. Blenkharn JI. Prevention of septic complications associated with TPN. JPEN J Parenter Enteral Nutr. 1986;10:436-437. doi: 10.1177/0148607186010004436.

13. Bisseling TM, Willems MC, Versleijen MW, Hendriks JC, Vissers RK, Wanten GJ. Taurolidine lock is highly effective in preventing catheter-related bloodstream infections in patients on home parenteral nutrition: a heparin controlled prospective trial. Clin Nutr. 2010;29:464-8. doi: 10.1016/j. clnu.2009.12.005.

14. Dümichen MJ, Seeger K, Lode HN, Kühl JS, Ebell W, Degenhardt P, et al. Randomized controlled trial of taurolidine citrate versus heparin as catheter lock solution in pediatric patients with haematological malignancies. J Hosp Infect. 2012;80:304-9. doi: 10.1016/j.jhin.2012.01.003.

15. Al Malki H, Othman M, Osman B, Rashid A, Fituri O, Asim M. TauroLock effectively reduces tunneled dialysis 
catheter-related infection and catheter exchange rates among hemodialysis patients. Open J Nephrol. 2016;6:55.

16. Fontseré N, Cardozo C, Donate J, Soriano A, Muros $\mathrm{M}$, Pons $\mathrm{M}$, et al. Tunneled catheters with taurolidinecitrate-heparin lock solution significantly improve the inflammatory profile of hemodialysis patients. Antimicrob Agents Chemother. 2014;58:4180-4.

17. Stein J. A randomized, controlled trial of taurolidineheparin-citrate locks in prevention of recurrence of catheter-related bacteremia in hemodialysis Patients. 50th Congress of the European Renal Association-European Dialysis and Transplant Association (ERA-EDTA); 2013; Istanbul, Turkey; SO063.

18. Zwiech R, Adelt M, Chrul S. A Taurolidine-CitrateHeparin Lock Solution Effectively Eradicates Pathogens From the Catheter Biofilm in Hemodialysis Patients. Am J Ther. 2016;23:e363-8.

19. Solomon LR, Cheesbrough JS, Bhargava R, Mitsides N, Heap M, Green G, et al. Observational Study of need for thrombolytic therapy and incidence of bacteremia using taurolidine-citrate-heparin, taurolidine-citrate and heparin catheter locks in patients treated with hemodialysis. Semin Dial. 2012;25:233-8. doi: 10.1111/j.1525139X.2011.00951.x.

20. Liu J, Wang CA, Zhao H, Zhang J, Ma J, Hou Y, et al.
Anticoagulant therapies versus heparin for the prevention of hemodialysis catheter-related complications: systematic review and meta-analysis of prospective randomized controlled trials. Int J Clin Exp Med. 2015;8:11985-95.

21. Murray EC, Deighan C, Geddes C, Thomson PC. Taurolidine-citrate-heparin catheter lock solution reduces staphylococcal bacteremia rates in hemodialysis patients. QJM. 2014;107:995-1000. doi: 10.1093/qjmed/hcu128.

22. Kavosi Z, Khorrami MS, Keshavarz K, Jafari A, Meshkini $\mathrm{AH}$, Safaei HR, et al. Is Taurolidine-citrate an effective and cost-effective hemodialysis catheter lock solution? A systematic review and cost-effectiveness analysis. Med J Islam Repub Iran. 2016;30:347.

23. Solomon LR, Cheesbrough JS, Ebah L, Al-Sayed T, Heap M, Millband N, et al. A randomized double-blind controlled trial of taurolidine-citrate catheter locks for the prevention of bacteremia in patients treated with hemodialysis. Am J Kidney Dis. 2010;55:1060-8. doi: 10.1053/j. ajkd.2009.11.025.

24. Liu Y, Zhang AQ, Cao L, Xia HT, Ma JJ. Taurolidine lock solutions for the prevention of catheter-related bloodstream infections: a systematic review and meta-analysis of randomized controlled trials. PLoS One. 2013;8:e79417. doi: 10.1371/journal.pone.0079417.

Copyright (c) 2018 The Author(s); Published by Nickan Research Institute. This is an open-access article distributed under the terms of the Creative Commons Attribution License (http://creativecommons.org/licenses/by/4.0), which permits unrestricted use, distribution, and reproduction in any medium, provided the original work is properly cited. 